\title{
Transgenic Expression of the Human Amphiregulin Gene Induces a Psoriasis-like Phenotype
}

\author{
Paul W. Cook, ${ }^{\star}$ Michael Piepkorn, ${ }^{\ddagger}$ Christopher H. Clegg, ${ }^{\S}$ Gregory D. Plowman, J. Mark DeMay, ${ }^{\star}$ Jeffrey R. Brown, ${ }^{\star}$ \\ and Mark R. Pittelkow $\llbracket$ \\ *Department of Dermatology and Department of Cell and Developmental Biology, The Oregon Health Sciences University, Portland, \\ Oregon 97201; ${ }^{\ddagger}$ Division of Dermatology, University of Washington School of Medicine, Seattle, Washington 98195; ${ }^{\circledR}$ Bristol-Myers \\ Squibb Pharmaceutical Research Institute, Seattle, Washington 98121; "Sugen Corporation, Redwood City, California 94063; and \\ "Department of Dermatology, Department of Biochemistry and Molecular Biology, Mayo Clinic/Foundation, Rochester, Minnesota 55905
}

\begin{abstract}
Amphiregulin (AR) is a heparin-binding, heparin-inhibited member of the epidermal growth factor (EGF) family and an autocrine growth factor for human keratinocytes. Previous studies have shown that AR expression is increased in psoriatic epidermis. To test the hypothesis that aberrant AR expression is central to the development of psoriatic lesions, we constructed a transgene (K14-ARGE) encoding a human keratin 14 promoter-driven AR gene. Our results indicate that transgene integration and subsequent expression of AR in basal keratinocytes correlated with a psoriasis-like skin phenotype. Afflicted mice demonstrated shortened life spans, prominent scaling and erythematous skin with alopecia, and occasional papillomatous epidermal growths. Histologic examination revealed extensive areas of marked hyperkeratosis with focal parakeratosis, acanthosis, dermal and epidermal lymphocytic and neutrophilic infiltration, and dilated blood vessels within the papillary dermis. Our results reveal that $A R$ exerts activity in the skin that is distinct from that of transgenic transforming growth factor- $\alpha$ or other cytokines, and induces skin pathology with striking similarities to psoriasis. Our observations also link the keratinocyte EGF receptor-ligand system to psoriatic inflammation, and suggest that aberrant expression of AR in the epidermis may represent a critical step in the development or propagation of psoriatic lesions. (J. Clin. Invest. 1997. 100:2286-2294.) Key words: growth factor • inflammation • skin $\cdot$ proliferation
\end{abstract}

\section{Introduction}

Psoriasis is an inflammatory and epidermal hyperproliferative skin disease that affects $\sim 2 \%$ of the population. The clinical manifestation of this disease is characterized by erythematous scaling papules and plaques that can cover a large percentage of the skin surface, and is sometimes accompanied by an ar-

Some of this material has been presented in abstract form (1997. $J$. Investig. Dermatol. 108:560A).

Address correspondence to Dr. Paul W. Cook, Department of Dermatology L 468, The Oregon Health Sciences University, Portland, OR 97201. Phone: 503-220-8262 ext. 6661; FAX: 503-721-7953; E-mail: cookp@ohsu.edu

Received for publication 8 June 1997 and accepted in revised form 29 August 1997.

The Journal of Clinical Investigation

Volume 100, Number 9, November 1997, 2286-2294

http://www.jci.org thritic condition. Although the etiology of psoriasis has not been elucidated, the development of psoriatic skin lesions has been shown to be associated with infiltration of cells from the immune system, including $\mathrm{T}$ lymphocytes, macrophages, and neutrophils. The aberrant activity of activated $\mathrm{T}$ lymphocytes is thought to be paramount in the pathogenesis of psoriasis, as immunosuppressive agents such as methotrexate, cyclosporine, tacrolimus, IL-2 receptor-targeted toxins, and corticosteroids are efficacious for this disease (1-6). Furthermore, activated CD4-positive $\mathrm{T}$ lymphocytes from patients with psoriasis have been shown to initiate psoriatic lesion formation in uninvolved human psoriatic skin grafted onto SCID/SCID (severe combined immunodeficient $)^{1}(7,8)$, and SCID/SCID mice reconstituted with naive CD4-positive $\mathrm{T}$ lymphocytes possessing a minor histocompatibility mismatch have been reported recently to initiate a psoriatic-like cutaneous reaction (9). There have also been several reports of hematopoietic stem cell transfer resulting in either the resolution or development of the psoriatic phenotype in humans (10-13). Although recent investigations have suggested that IFN- $\gamma$ or other uncharacterized factors derived from activated lesional $\mathrm{T}$ lymphocytes may selectively activate lesional keratinocyte proliferation (14), little is known regarding the mechanism by which infiltrating $\mathrm{T}$ lymphocytes are recruited into skin and subsequently activate psoriatic keratinocyte proliferation.

Amphiregulin (AR) and heparin-binding EGF-like growth factor (HB-EGF) are polypeptide growth factors of the EGF family that were isolated initially from the conditioned medium of human MCF-7 breast tumor cells and monocyte-like U937 cells, respectively $(15,16)$. These EGF-related growth factors are heparin-binding members of the EGF family and possess properties distinct from those of either TGF $\alpha$ or EGF. Collectively, these properties include affinity for heparin and heparin-like molecules $(16,17)$, heparin-dependent stimulation or inhibition of mitogenic activity (17-21), nuclear targeting signals and nuclear localization (16, 22-27), cell densitydependent responsiveness (17), and, unexpectedly, the ability of rat AR to mediate nuclear targeting-dependent mitogenic activity in the absence of EGF receptor tyrosine kinase activity (27). Additionally, we have demonstrated previously that the heparin-dependent modulation of AR- and HB-EGF-dependent mitogenic activity is mediated by the ability of heparin to either inhibit or enhance the interaction of these growth factors with EGF receptors $(17,20,21)$.

1. Abbreviations used in this paper: AR, amphiregulin; ARGE, amphiregulin gene; $\mathrm{H}$ and $\mathrm{E}$, hematoxylin and eosin; HB-EGF, heparinbinding EGF-like growth factor; K14, keratin 14; SCID, severe combined immunodeficient. 
We, along with other investigators, have shown that the heparin-binding members of the EGF family are involved in the autocrine growth of cultured human epidermal keratinocytes (17-19, 28-31). Among these growth factors, AR and HBEGF have emerged as likely mediators of autocrine signaling in human keratinocytes through interaction with EGF receptors expressed by these cells. Quantitatively, AR may account for most of the autocrine growth capacity of cultured keratinocytes, as HB-EGF- and TGF- $\alpha$-neutralizing antibodies block $<30 \%$ of the autocrine-driven keratinocyte proliferation $(30,31)$, while AR-neutralizing antibodies and heparin block 70 and $100 \%$ of this autocrine proliferation, respectively $(17,18,28,29)$. The expression of AR and HB-EGF in normal epidermis is typically low to undetectable $(25,26,32-35)$, but establishing keratinocytes in culture results in their conversion to an autonomous AR-driven phenotype. The mechanisms that mediate this cell culture-dependent mitogenic activation have not been elucidated.

Expression of AR has been shown to correlate with epidermal proliferation during cutaneous development in humans (25), and both AR and HB-EGF are upregulated after skin removal and organ culture ex vivo (35), whereas HB-EGF has been detected in porcine wound fluid (36). Similarly, elevation of AR expression has been observed in mice after tape stripping (37). Several studies, including our own, have also demonstrated that the EGF ligand-receptor pathway appears to be activated aberrantly in psoriatic epidermis and other epidermal proliferative pathologies via the expression of keratinocyte autocrine-acting mitogens (HB-EGF, TGF- $\alpha$, and $A R)$ and the EGF receptors to which these ligands bind $(26,32-35,38-40)$. As a result of these cumulative observations, we have speculated that AR and HB-EGF expression may be critical in orchestrating epidermal growth and development in both normal and pathological states, such as psoriasis.

Over the last several years, transgenic mice have been generated that epidermally target factors shown to be overexpressed in psoriatic lesions. These factors include the keratinocyte mitogen TGF- $\alpha$ and keratinocyte growth factor, the inflammatory cytokines IL-6, IL- $1 \alpha$ and its receptor, TNF- $\alpha$, and IFN- $\gamma$, and inflammatory costimulatory and adhesion molecules such as B7-1 and intercellular adhesion molecule-1 (41-50). Although expression of some of these gene products resulted in the stimulation of keratinocyte proliferation and subsequent hyperkeratosis or papilloma formation, or promoted cutaneous inflammation with leukocytic infiltrate and edema, none of these factors has been shown to produce a phenotype with all of the features of psoriasis. More recent investigations have demonstrated that the targeted expression of two unrelated gene products (i.e., bone morphogenetic protein and integrins) to the murine epidermis results in phenotypic changes similar to psoriasis $(51,52)$. It was speculated that the inappropriate expression of these diverse gene products leads to the disruption of the keratinocyte differentiation process and subsequent stimulation of not only keratinocyte hyperproliferation, but also inflammatory cell infiltration. In the preliminary investigations presented here, we overexpressed $A R$ in murine epidermis to determine whether the exaggerated expression of this EGF-related autocrine factor could initiate alterations in keratinocyte proliferation and differentiation, and induce the development of a hyperproliferative and inflammatory skin pathology that mimics psoriasis.

\section{Methods}

Transgenic expression vector and generation of transgenic mice. A human placental genomic library cloned into the Lambda FIX II vector (Stratagene Inc., La Jolla, CA) was screened for sequences specific to the human keratin 14 (K14, 50-kD keratin-1) gene (53). A 3,137-bp HindIII fragment beginning 2,342 bp upstream of the start codon for human K14 (GenBank accession numbers U11076 and J00124) was isolated, and the sequence was verified. A 2,337-bp fragment containing the K14 promoter/enhancer was reconstructed in the pBluescript II SK+ vector (Stratagene Inc.) from the upstream HindIII site to an artificial XbaI site created 6 bp upstream of the K14 start codon. This ligation generated unique XbaI, SpeI, and BamHI sites $3^{\prime}$ of the K14 promoter. A 0.63-kb BamHI/EcoRI fragment encoding a translational stop codon and the polyadenylation signal of human growth hormone was cloned into this recombinant vector and juxtapositioned in the forward orientation 3' to the K14 promoter. The entire AR coding region is contained on a $9.7-\mathrm{kb}$ SmaI/EcoRV fragment (54) spanning 6 exons and flanked by a $164-$ bp 5' and a 27-bp 3' untranslated sequence. This 9.7-kb AR genomic fragment was blunt-end ligated in the $5^{\prime}-3^{\prime}$ orientation into a unique BamHI site between the K14 promoter and stop-polyadenylation signal regions, and the corresponding expression vector was designated pK14-ARGE (amphiregulin gene). A linearized K14-ARGE transgene was generated by restriction digestion of pK14-ARGE with SalI and NotI, followed by purification via agarose gel electrophoresis, electroelution, and elutip anion exchange chromatography. The linearized K14-ARGE transgene was buffer-exchanged in ultrapure buffer containing $10 \mathrm{mM}$ Tris-base (pH 7.5) and $0.2 \mathrm{mM}$ EDTA, and adjusted to a concentration of $1 \mathrm{ng} / \mu \mathrm{l}$. The transgene was microinjected into the pronuclei of fertilized oocytes corresponding to FVB/ NCrIBR mouse strain (Charles River Laboratories, Wilmington, MA) and implanted into pseudopregnant surrogate mothers to produce putative founder mice expressing the K14-ARGE transgene. Approximately 50 pups were produced for screening. A female K14ARGE-expressing founder mouse was back-bred into the FVB/ NCrIBR mouse strain to generate F1 progeny. Mice were housed under specific-pathogen-free conditions, moved to separate cages upon weaning, and killed, when indicated, by $\mathrm{CO}_{2}$ asphyxiation.

$P C R$ analysis of genomic DNA. Tail clippings were obtained from both founder and F1 litters, and genomic DNA was isolated by proteinase $\mathrm{K}$ digestion, phenol/chloroform extraction, and ethanol precipitation. $1 \mu \mathrm{g}$ of genomic DNA was subjected to PCR analysis using forward (CACACCTCCCCCTGTGAATCAC) and reverse (GAATCCTCACCTGAGCCGAGTATC) oligonucleotides specific for the human K14 promoter and the first exon of human AR (54), respectively. The PCR reaction conditions consisted of a 5-min step at $91^{\circ} \mathrm{C}$ to denature the sample and melt AmpliWax beads (Roche Molecular Systems, Inc., Nutley, NJ), and a room-temperature cooling step to form a wax interface between lower and upper reactants. Before temperature cycling, a $2-\min 94^{\circ} \mathrm{C}$ denaturization step was performed to mix upper and lower reactants (hot start), and 30 cycles of amplification were performed $\left(94^{\circ} \mathrm{C}, 1 \mathrm{~min} ; 59^{\circ} \mathrm{C}, 1 \mathrm{~min} ; 72^{\circ} \mathrm{C}, 2 \mathrm{~min}\right)$.

This protocol was demonstrated to amplify a predicted transgenespecific 531-bp PCR product corresponding to a region encompassing the $3^{\prime}$ of the human K14 promoter, the internal cloning vector sequence, and the $5^{\prime}$ end of human AR exon 1 (54). As a positive control, forward (CTCAGGCTGCTGTACTGGTTCTTG) and reverse (ACGACCCCCTTTTTTTGGAGAC) primers corresponding to the endogenous mouse plasminogen activator inhibitor-1 gene were used under identical PCR reaction conditions to amplify a predicted 456bp PCR product. PCR products were subjected to electrophoresis through $1.1 \%$ agarose gels containing ethidium bromide and visualized via UV illumination. Gels were photographed and scanned to create a digital image of the amplification products. Images were assembled for the preparation of figures, making no adjustments to either contrast or brightness.

Histopathology. Mouse skin tissue was fixed in $10 \%$ formalin 
PBS, paraffin-embedded, sectioned $(5 \mu \mathrm{m})$, washed, and stained with hematoxylin and eosin ( $\mathrm{H}$ and $\mathrm{E})$ for the microscopic characterization of transgene-associated histopathologic changes.

Immunohistochemistry. Mouse skin was fixed in methyl-Carnoy's solution and embedded in paraffin. Skin was sectioned $(6.5 \mu \mathrm{m})$ and mounted on superfrost glass slides for processing. For immunodetection of human AR protein in transgenic mouse skin, tissue was deparaffinized in xylene, blocked with $5 \%$ normal goat serum, incubated for $60 \mathrm{~min}$ with the $10 \mu \mathrm{g} / \mathrm{ml}$ 6R1C mouse anti-human AR mAb (18, $25,26)$ in $5 \%$ BSA, as described previously for immunolocalization of AR in human skin pathologies (26). After washing, this step was followed by a 30 -min incubation with multiple-adsorption biotinylated secondary goat anti-mouse IgG (12062D; PharMingen, San Diego, CA) antibody (1:200) in the presence of 5\% BSA. After washing, endogenous peroxidase activity was blocked by incubating mounted tissue with $1.5 \% \mathrm{H}_{2} \mathrm{O}_{2}$ in methanol for $10 \mathrm{~min}$. Specific interaction of the $6 \mathrm{R} 1 \mathrm{C}$ antibody with human AR was visualized by washing the slides and subsequently incubating the tissue with the avidin-biotinperoxidase complex and diaminobenzidine reagents (PK-6100 and SK-4100; Vector Laboratories, Inc., Burlingame, CA), as described by the manufacturer. All washes and incubations were carried out in $0.1 \mathrm{M}$ Tris/normal saline ( $\mathrm{pH} 7.5)$ at room temperature.

Similar protocols were used to detect the murine pan T lymphocyte CD3 and cell cycle-specific Ki67 markers in transgenic mouse skin. For detection of $\mathrm{T}$ lymphocytes, tissue was unmasked in $1 \mathrm{mM}$ EDTA ( $\mathrm{pH} \mathrm{8.0)}$ at $94^{\circ} \mathrm{C}$ for $6 \mathrm{~min}$, followed by a 30 -min cool-down period at room temperature. Tissue was blocked subsequently with $5 \%$ normal rabbit serum and incubated with a primary rat anti-murine CD3 mAb (NCL-CD3-12; Novocastra Laboratories, Newcastle upon Tyne, UK) at a 1:10 dilution. This step was followed by incubation with mouse-adsorbed biotinylated secondary rabbit anti-rat IgG (BA4001; Novocastra Laboratories) antibody at a 1:50 dilution. For detection of Ki67, tissue was unmasked in $10 \mathrm{mM}$ sodium citrate (pH 6.0) at $94^{\circ} \mathrm{C}$ for $6 \mathrm{~min}$, followed by a 30-min cool-down period at room temperature. The tissue was then blocked with $5 \%$ normal goat serum and incubated with a 1:25 dilution of mouse anti-murine Ki67 mAb (NCL-Ki67-MM1; Novocastra Laboratories). This step was followed by incubation with multiple-adsorption biotinylated secondary goat anti-mouse IgG (12062D; PharMingen) antibody used at a 1:200 dilution. For negative controls, all immunohistochemistry experiments were also performed in the presence of equal concentrations of isotype-matched nonspecific antibodies as a substitute for the primary antibody.

\section{Results}

Generation of K14-ARGE transgenic mice. The vector conferring human K14 promoter/enhancer-driven expression of the human AR gene, pK14-ARGE, was used to generate a linearized transgene for creation of both founder and F1 progeny mice (Fig. 1). The candidate founders and F1 progeny were screened for integration of K14-ARGE by PCR analysis. Fig. 2 displays some of the results of this analysis, and includes the first phenotypically altered founder (Founder-1), a nontransgenic mouse (Normal), two phenotypically unaffected littermates (Littermate-1 and Littermate-2), one phenotypically affected F1 derived from the first founder, a phenotypically altered second founder (Founder-2), a third phenotypically affected founder (Founder-3), and two additional normal littermates (Littermate-3 and Littermate-4). 5 of the 50 pups from the transgenic litters were genopositive for the K14-ARGE transgene, and all except one of these animals displayed some degree of skin pathology. Thus, K14-ARGE transgene integration correlated strongly with phenotypically altered skin in these mice.

Five K14-ARGE-positive founders were generated, and

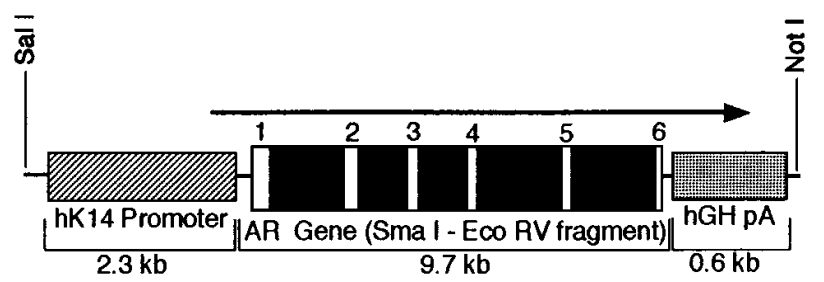

Figure 1. K14-ARGE transgenic expression vector. Diagram of K14ARGE transgenic expression vector depicting the human K14 ( $h K 14$ ) promoter, the human AR gene, including 6 exons encoding the AR transcript and primary translation product, and the human growth hormone polyadenylation-stop signal $(h G H p A)$ sequences.

three possessed profound phenotypic alterations of the skin, whereas the fourth and fifth K14-ARGE-positive founder mice displayed mild or no detectable aberrant skin phenotype, respectively. The diminished or absent phenotypes of founder4 and -5 may have been due to integration of transgene only in tissues that do not promote expression through the K14 promoter (mosaicism), integration of transgenes at genomic sites that inactivate the expression of the transgene, or integration of defective (mutated) transgenes that no longer express gene product in the target tissue. Founder- 1 (female, not pictured in Fig. 3) survived $\sim 5$ mo and produced several litters that included individuals exhibiting a psoriasiform skin phenotype. Affected F1 pups from this founder were runted and did not survive beyond 2-3 wk. Both founder-1 and affected F1 progeny showed a dramatic psoriasis-like cutaneous phenotype that included areas of scaling and erythematous skin. Alopecia, occasional papillomatous growths, and edema were also observed. Formalin-fixed representative specimens of male 2-wk-old normal and phenotypically affected F1 littermates from founder- 1 are shown in Fig. $3 A$. The psoriasis-like phenotype was strikingly evident in a 3 -wk-old third female founder (founder-3), shown in Fig. 3, $B-D$.

Epidermis-specific expression of $A R$ in K14-ARGE transgenic mice. In an attempt to establish a correlation between expression of protein from the AR transgene and the aberrant phenotype in K14-ARGE mice, fixed skin tissue from the ear of the phenotypically affected founder- 3 and its normal litter-

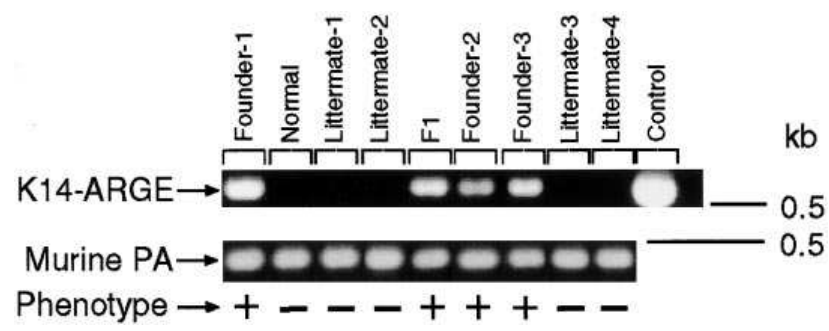

Figure 2. PCR analysis of founder and F1 K14-ARGE transgenic mice. Genomic DNA was subjected to PCR analysis as described in Methods. Top, Amplification (531 bp) with primers specific for the K14-ARGE transgene. Normal and control, Amplification from the genomic DNA of a normal nontransgenic mouse and $1 \mathrm{ng}$ of linearized K14-ARGE transgene, respectively. Bottom, Amplification (456 bp) of the same genomic DNA samples with primers specific for the endogenous murine plasminogen activator $(P A)$ inhibitor-1 gene as internal positive control. 

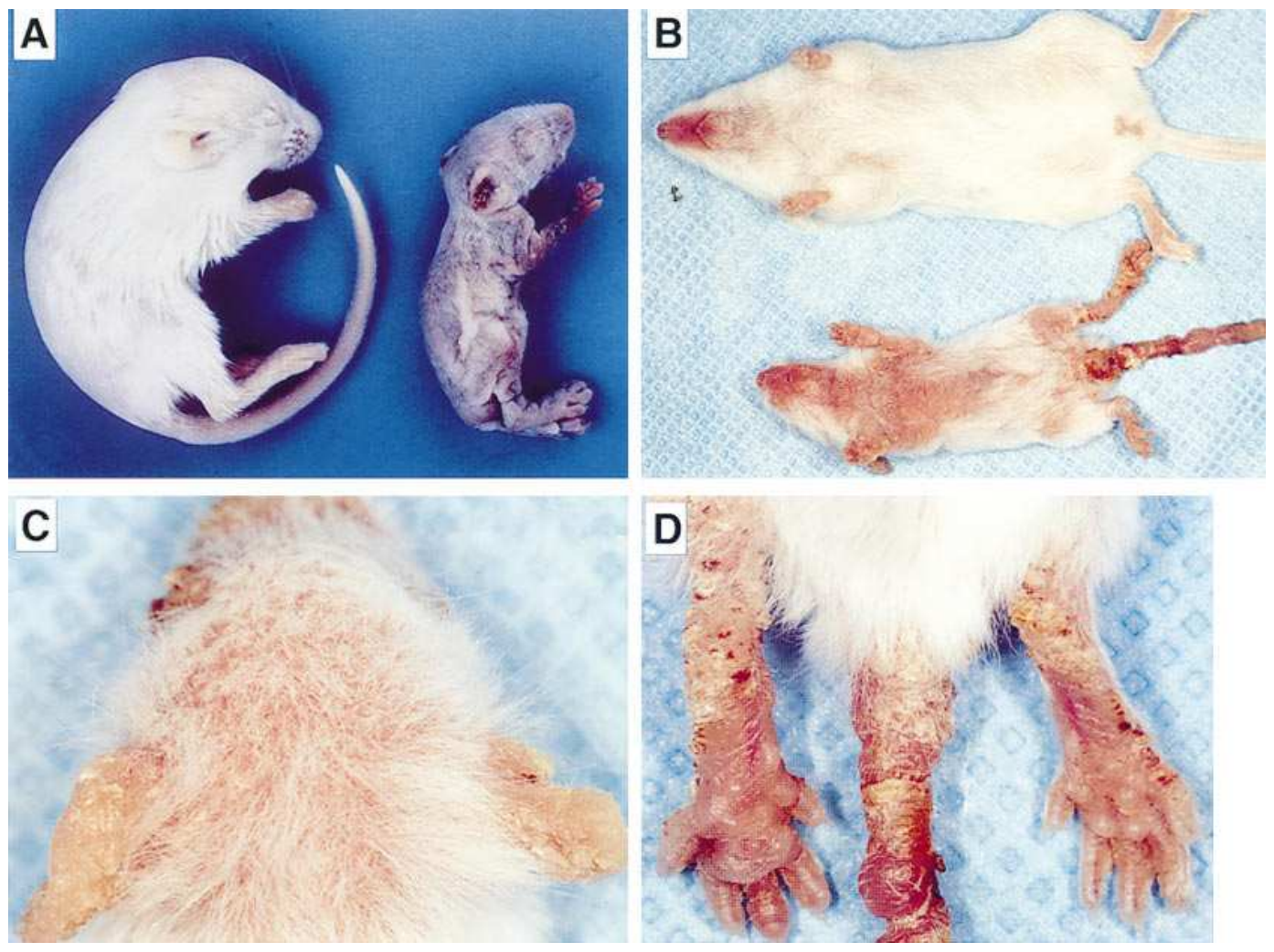

Figure 3. Gross macroscopic phenotype of founder and F1 K14ARGE transgenic mice. (A) 2-wk-old normal male littermate (left), and 2-wk-old phenotypically affected $\mathrm{F} 1$ male from founder-1 (right; founder-1 not pictured). (B) Ventral view of 3-wk-old female normal littermate (top), and 3-wk-old phenotypically affected female founder-3 (bottom). (C) Dorsal view, head and ears, of founder-3. $(D)$ Dorsal view, tail and legs, of founder-3. mate (shown in Fig. $3 B$ ) were examined for expression of immunoreactive human AR protein (Fig. 4, $A-C$ ). The results demonstrate that AR immunoreactivity was found in the cytoplasm and localized to both basal and some suprabasal keratinocytes of the phenotypically affected K14-ARGE mouse (Fig. $4 B$ ), whereas human AR-specific immunoreactivity was undetectable in the phenotypically unaffected normal littermate (Fig. $4 \mathrm{~A}$ ). The AR protein was not present uniformly over the entire epidermis of founder-3, and we speculate that this discontinuous expression could be due to mosaic expression of the K14-ARGE transgene in this animal. Collectively, these data suggest that human AR expression in the basal and suprabasal epidermis correlates with the psoriasis-like cutaneous phenotype of the K14-ARGE transgenic mouse.
Histopathology of skin derived from normal and K14$A R G E$ transgenic mice. The dermatopathology of phenotypically affected founder K14-ARGE mice was examined by $\mathrm{H}$ and $\mathrm{E}$ staining of formalin-fixed tissue from two different founder mice. Fig. 5 displays the results of this microscopic examination of the tissues from the ear and tail of normal (Fig. 5, $A$ and $D$ ) and phenotypically affected (Fig. 5, $B, C$, and $E-I$ ) founder K14-ARGE mice. Histological examination of the ear from the founder-3 K14-ARGE mouse revealed alterations resembling those observed in human psoriatic skin, including characteristic findings of severe hyperkeratosis, cellular acanthosis, increased frequency of mitotic cells in the basal epidermis, segmental parakeratosis, and a dermal cellular infiltrate showing the morphologic features of lymphocytes (Fig. 5, B
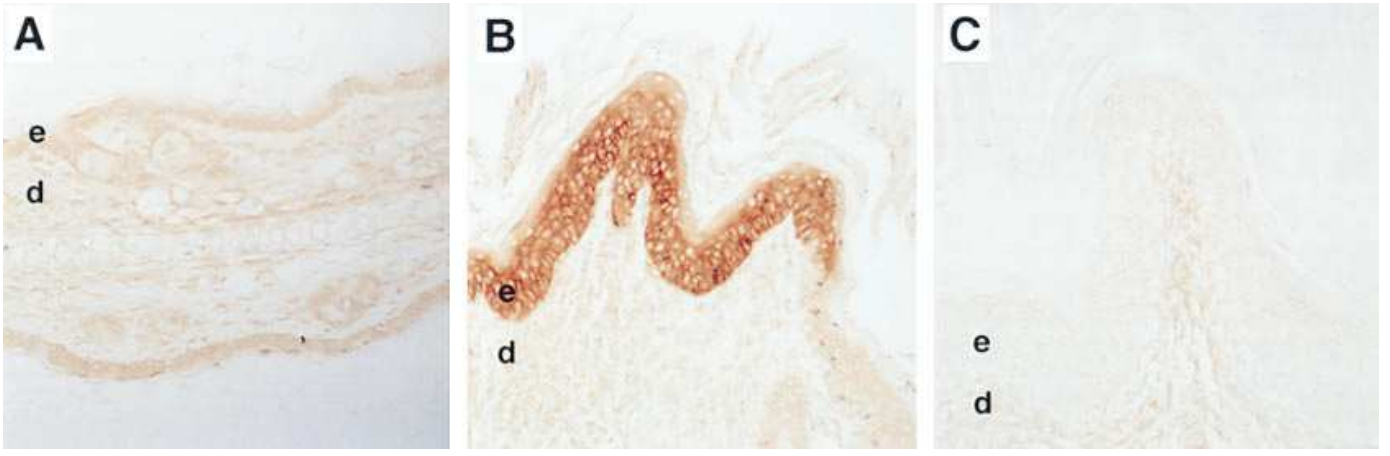

Figure 4. Expression of AR protein in K14-ARGE transgenic mice. $(A$ and $B) 6 \mathrm{R} 1 \mathrm{C}$ immunoperoxidasestained ear from normal littermate and founder-3, respectively. $(C)$ Immunoperoxidase-stained ear from founder-3 incubated with isotype-matched irrelevant mAb. All photomicrographs were created from scanned digital images of color 35-mm slides photographed at a magnification of 50.e, Epidermis. $d$, Dermis. 

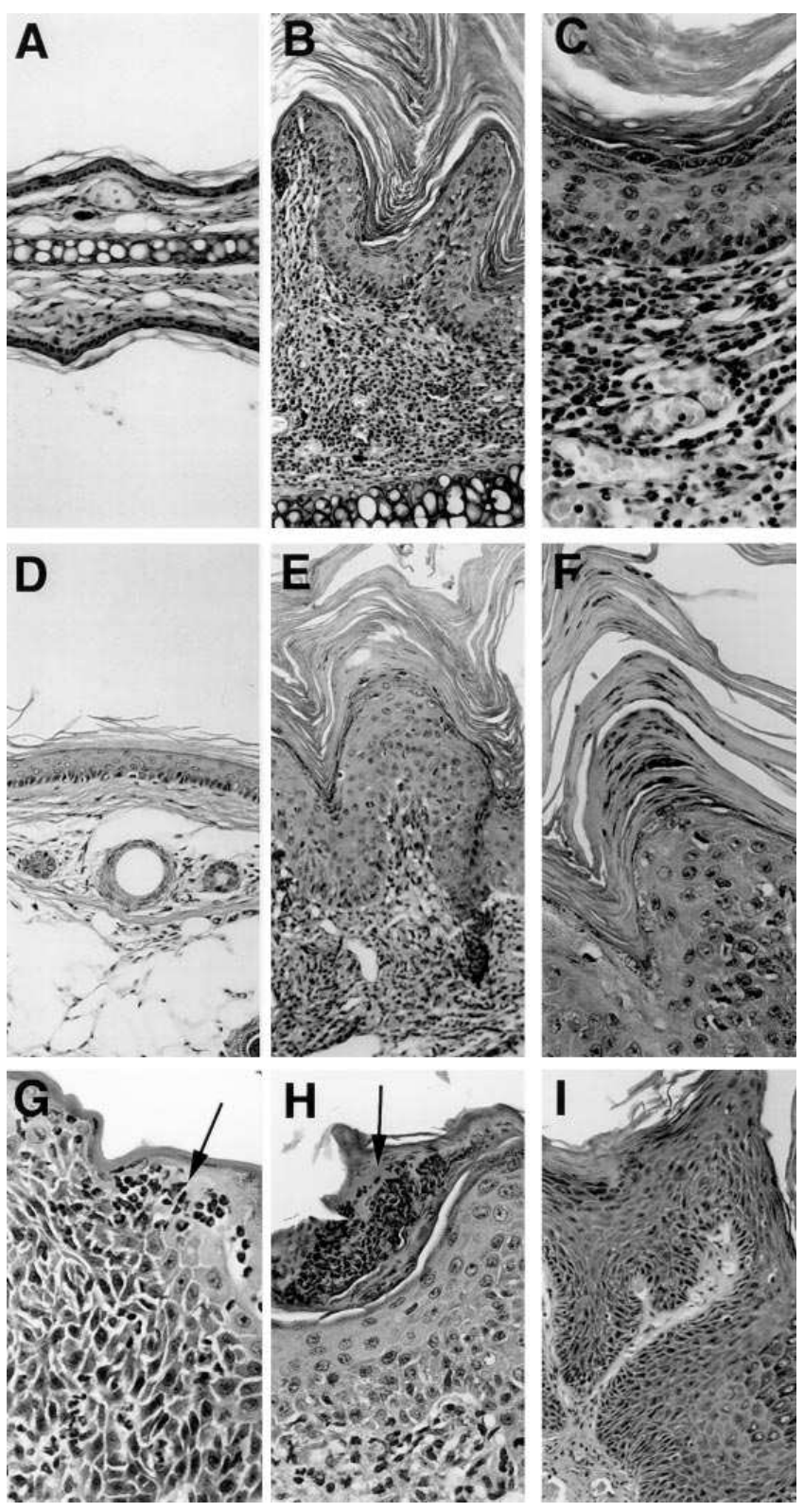

Figure 5. Histopathology of founder K14-ARGE transgenic mice. Tissue was processed for $\mathrm{H}$ and $\mathrm{E}$ staining as described in Methods. (A) Ear from normal littermate to founder-3. (B) Ear from founder-3. $(C)$ Ear from founder-3 at higher magnification. $(D)$ Tail from normal littermate to founder-2. (E) Tail from founder-2. $(F)$ Tail from founder-2 at higher magnification. $(G)$ Ear from founder-2, depicting spongiotic pustule (arrow). (H) Ear from founder-2, depicting microabscess (arrow). (I) Papillomatous epidermal growth from founder-3 tail. All photomicrographs were converted to grayscale from scanned digital images of color 35-mm slides photographed at magnifications of $50(A, B, D, E$, and $I)$ or $100(C, F, G$, and $H)$.

and $C$ ) that tended to cluster around tortuous, dilated blood vessels (Fig. $5 \mathrm{C}$ ). The dermal infiltrate also included some cells with histological characteristics of neutrophils and histiocytes. Additionally, the histology revealed that the hair follicles of the affected founders and F1 progeny were generally less numerous and more poorly developed in these AR-expressing mice. This observation was strikingly evident in severely affected areas where hair follicles appeared to be absent.

Histological examination of skin from a different anatomical location (tail) of a second founder mouse (3-wk-old female, not pictured in Fig. 3) revealed similar profound changes, but also exhibited more numerous parakeratotic nuclei within a hyperkeratotic stratum corneum, and more prominent neutrophilic exocytosis, which could be observed focally in all layers of the epidermis (Fig. 5, $E$ and F). Other features commonly observed in psoriatic lesions included a reduced granular layer at sites of parakeratosis, neutrophils in the parakeratotic stratum corneum, spongiotic pustules, and microabscesses (Fig. 5, $F-H$, respectively). As observed grossly (Fig. $3 D$ ), the phenotypically affected mice occasionally displayed papillomatouslike growths that by histological examination showed discrete acanthoma-like epidermal changes with minimal inflammatory infiltrate (Fig. 5 I).

Detection of proliferative (Ki67) and pan T lymphocytespecific antigen (CD3) in K14-ARGE transgenic mice. To demonstrate correlation between AR expression in the epidermis and increased epidermal keratinocyte proliferation (hyperproliferation) in K14-ARGE mice, fixed skin tissues from the ears of the phenotypically affected founder-3 mouse and a normal littermate (pictured in Fig. $3 \mathrm{~B}$ ) were examined for expression of the proliferative (cell cycle-specific) marker Ki67 by immunohistochemistry (Fig. 6, $A-C$ ). Ki67 immunoreactivity was expressed by numerous basal keratinocytes of the phenotypically affected K14-ARGE mouse (Fig. 6 B), whereas this immunoreactivity was observed rarely in the normal littermate (Fig. $6 \mathrm{~A}$ ). These results suggest that human AR expression in the basal epidermis results in markedly increased epidermal cell proliferation.

To begin to characterize the cutaneous inflammatory cell response of the K14-ARGE transgenic mice, fixed skin tissue from the ears of the mice characterized for AR expression were also examined for the presence of CD3-immunoreactive T lymphocytes (Fig. 6, $D-F$ ). The results demonstrate that the CD3 antigen localized predominantly to cells in the dermis bearing histological resemblance to lymphocytes (Fig. $6 \mathrm{E}$ ). CD3 immunoreactivity was also observed within the epidermis in proximity to the dermoepidermal junction of K14-ARGE mouse. In contrast, CD3 expression was observed infrequently in normal littermate skin (Fig. $6 \mathrm{D}$ ). These results suggest that human AR overexpression in the basal and suprabasal epidermis results in the recruitment of CD3-positive $\mathrm{T}$ lymphocytes into both the dermis and epidermis.

\section{Discussion}

Recent evidence implicates aberrant activation of the immune system and T lymphocyte localization in skin as critical components in the pathogenesis of psoriasis (1-14), but the etiologic $\operatorname{defect}(\mathrm{s})$ in this disease remain elusive. A role for upregulated expression of autocrine regulatory networks in the pathogenesis of psoriasis has emerged from previous investigations that have suggested that epidermal hyperplasia and aberrant differentiation may result from dysregulation of the epidermal keratinocyte EGF receptor-ligand system (26, 31-35, 38, 39). Elucidation of the critical molecular mechanisms underlying these changes may be ultimately useful in identifying novel and more effective therapeutic strategies in the treatment of psoriasis and other epidermal proliferative disorders. 


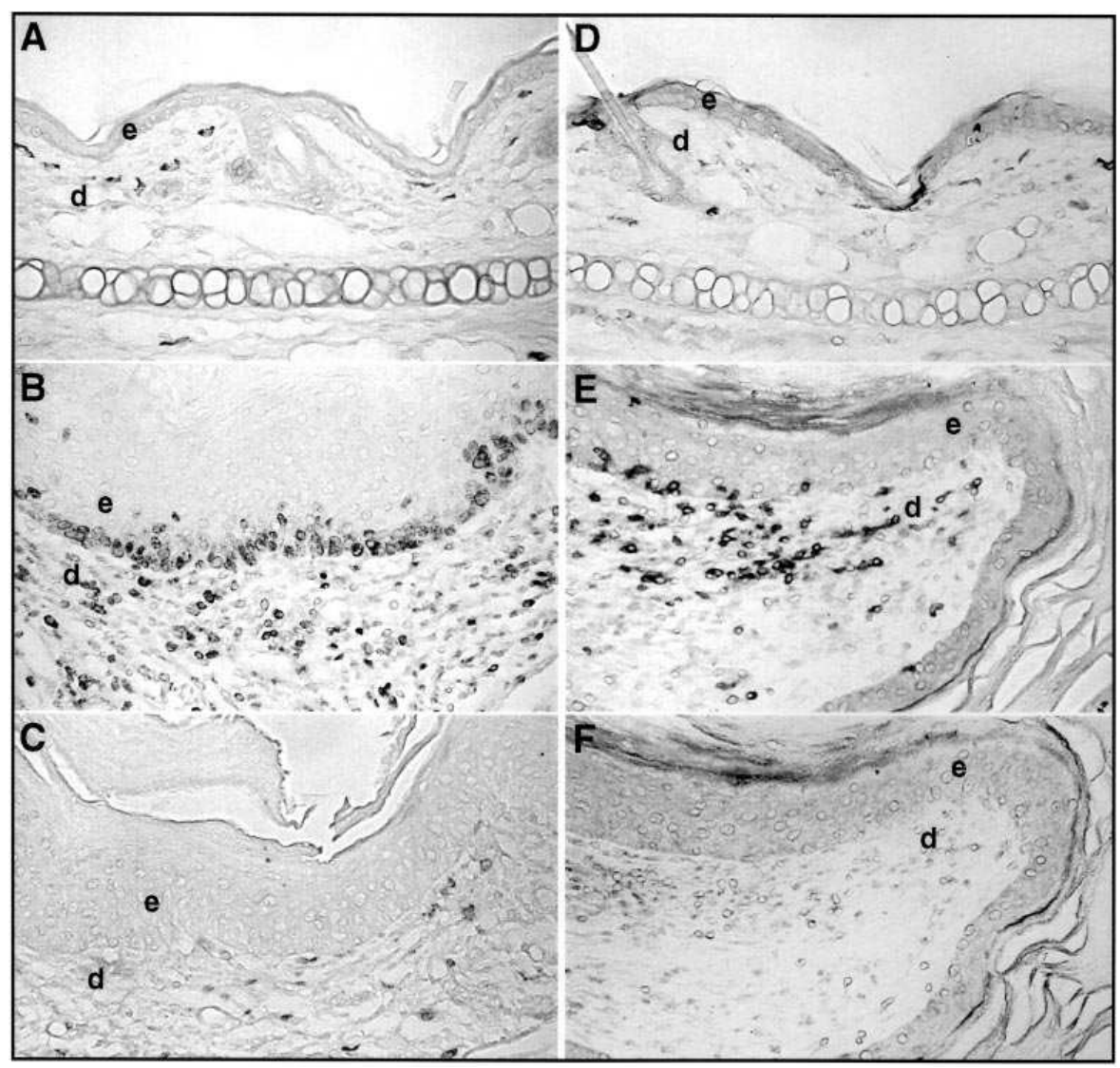

Figure 6. Detection of proliferative (Ki67) and pan T lymphocyte marker (CD3) expression in K14-ARGE transgenic skin. ( $A$ and $B)$ Ki67 immunoperoxidase-stained ear from normal littermate and founder-3, respectively. $(C)$ Immunoperoxidasestained ear from founder-3 incubated with isotype-matched irrelevant mAb. ( $D$ and E) CD3 immunoperoxidase-stained ear from normal littermate and founder-3, respectively. $(F)$ Immunoperoxidase-stained ear from founder-3 incubated with isotypematched irrelevant mAb. All photomicrographs were converted to grayscale from scanned digital images of color $35-\mathrm{mm}$ slides photographed at a magnification of 100. $e$, Epidermis. $d$, Dermis.
Theoretical mechanisms to account for the hyperplastic and perturbed differentiative phenotype of psoriatic epidermis include loss or suppression of growth inhibitors, autoinduction of growth factor receptors, and overexpression of autocrine growth factors (55). In vivo, overexpression of AR at both the mRNA and protein levels has been demonstrated in the cutaneous lesions of psoriasis (26, 33-35), and these collective observations suggest strongly a hypothetical role for AR in the pathophysiology of this disease. To test this hypothesis, we introduced a human AR genomic clone into mouse embryos and targeted its expression to the basal epidermis. Founder and F1 progeny were generated in order to characterize the gross and microscopic changes associated with overexpression of human AR in the mouse. The AR transgene was introduced successfully into the germline of at least one founder mouse and passed to F1 mice, as evidenced by PCR amplification of specific human K14-AR sequences from both affected founder-1 and F1 genomic DNA. Human AR protein was also expressed in the basal and suprabasal epidermal keratinocytes, and correlated with both transgene integration and the psoriasiform phenotype.

Grossly, the cutaneous phenotype of selected founder animals was characterized by confluent erythematous scaling, which was accentuated over the tail, feet, ears, ventral thorax, and snout. Papillomatous growths were evident focally on tail skin and at other sites prone to repetitive trauma, and there was also prominent patchy alopecia. At the microscopic level, affected epidermis displayed hyperkeratosis, focal parakerato- sis with concomitant thinning or absence of the stratum granulosum, acanthosis with some areas of papillomatosis, and infiltration of neutrophils into the spinous, granular, and cornified epidermal layers, producing spongiotic pustules and microabscesses. Dilatation of the superficial dermal microvascular channels was also evident, and there was an associated leukocytic inflammatory cell infiltrate in the adjacent dermis that was composed primarily of CD3-positive T lymphocytes, but included also cells resembling neutrophils and histiocytes. Exocytosis of CD3-positive T lymphocytes into the epidermis was also apparent.

When compared to psoriasis, this model demonstrates some histological differences, such as an increased presence of the epidermal granular layer, lack of pronounced rete ridge elongation, frequent papillomatosis, and a diminished presence of hair follicles. The absence of rete ridge elongation and presence of papillomatosis may not be unexpected, as papillomatosis is found in some forms of human psoriasis (rupioid). Because of structural differences between mouse and human skin (thinner mouse epidermis, diminished rete ridges), we and other investigators have speculated that the skin of psoriasis-like engineered mice $(9,52)$ may default to a papillomatous morphology, while human psoriatic skin evolves to morphology with elongated rete pegs. Thus, this characteristic of psoriasis might be due to the rete ridges and thicker epidermis acting as a template for rete elongation in humans. Although hair loss is not recognized readily in patients with psoriasis of the scalp, significant alopecia has been documented frequently 
and is well-accepted in cases of erythrodermic and pustular psoriasis, and studies have also shown that common plaque psoriasis demonstrates varying degrees of alopecia and/or hair shaft thinning (56-61). Furthermore, EGF infusion induces hair loss, and transgenic expression of TGF- $\alpha$ has been shown to correlate with alopecia as well as other hair growth abnormalities $(41,42,62)$. Because K14 has been shown to be expressed in the outer root sheath (63), alopecia is not surprising, since our transgenic model was generated using the K14dependent overexpression of a psoriasis-associated EGF-like ligand (AR). Collectively, our findings demonstrate that the targeted expression of human AR to the murine basal epidermis induces most of the common and several of the less common microscopic changes found in psoriasis.

Other possible explanations for the AR-dependent leukocytic infiltration seem unlikely, such as cutaneous infections, as specific stains did not reveal pathogenic organisms in the transgenic tissues. It is also unlikely that the transgenic mice are mounting a humoral- or cell-mediated response to human AR-expressing keratinocytes, as unactivated keratinocytes are not known to be efficient antigen-presenting cells, and the phenotypically affected transgenic animals displayed no evidence of basal keratinocyte degeneration or necrosis. Additionally, the transgenic mice should also be immunotolerant to human AR due to its K14 promoter-dependent expression before the development of the thymus, on day 9.5 of mouse fetal development (64).

Because the afflicted K14-ARGE F1s were runted and had markedly reduced longevity (2-3 wk), the generation of viable F1 offspring and subsequent establishment of an AR-expressing psoriasiform mouse line has not been possible. We speculate that the elevated expression of AR in other tissues (e.g., thymic and esophagogastric epithelium) may cause deleterious side effects that contribute to poor survival.

While the mechanisms directing the epidermal overexpression of AR in psoriasis remain undefined, our results are consistent with the hypothesis that overexpression of AR and possibly other related ligands (HB-EGF) mediates the cutaneous phenotype of psoriasis. Although the potential mechanisms for this response could include a direct proinflammatory effect of AR (Fig. 7, dashed arrow), we propose that these features of psoriasis may also represent AR-mediated upregulation of proinflammatory cytokine circuits in the epidermis (Fig. 7, bold solid arrows). This indirect mechanism is supported by recent studies that have also speculated that the targeted overexpression of two unrelated gene products in murine epidermis, bone morphogenetic protein and integrins, leads to the disruption of keratinocyte differentiation, the stimulation of proliferation, and the infiltration of $\mathrm{T}$ lymphocytes and neutrophils into both the dermis and epidermis $(51,52)$. However, by comparison, AR overexpression induces a phenotype that resembles psoriasis most closely of any transgenic mouse model reported to date. Thus, we speculate that AR expression in the epidermis of our transgenic mice leads to AR-stimulated signal transduction and consequent activation of keratinocytemediated proinflammatory pathways. Indeed, our results are consistent with a mechanism in which infiltrating $\mathrm{T}$ lymphocytes enhance AR production in the epidermis and subsequently induce keratinocyte activation, leading to further immigration of activated $\mathrm{T}$ lymphocytes as well as other inflammatory cells, and subsequent development of other pathogenic changes commonly observed in psoriasis. With respect to

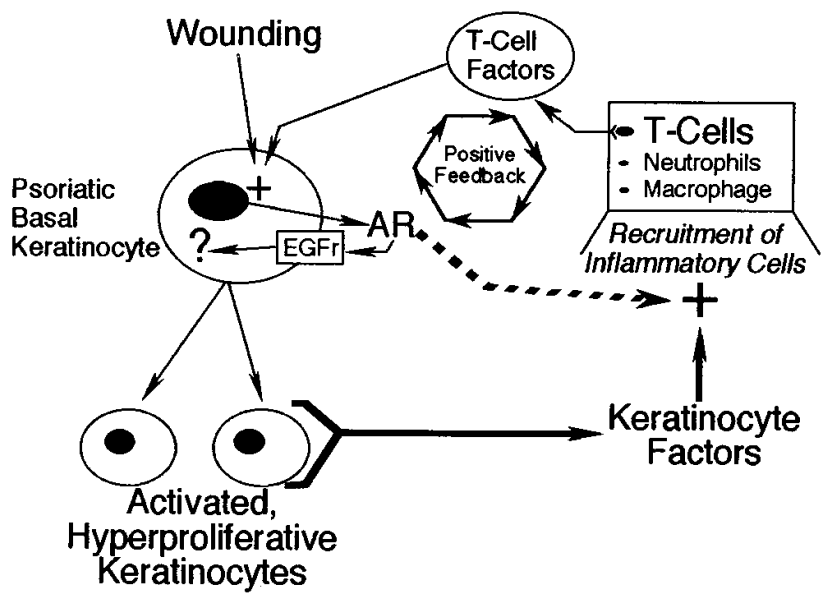

Figure 7. Model depicting epidermal AR expression and its putative role in the pathogenesis of psoriasis. EGFr, EGF receptor.

the recruitment of $\mathrm{T}$ lymphocytes, this mechanism would constitute a positive feedback loop and result in the rapid generation of fully developed psoriatic lesions (Fig. 7). It is also of interest that wounding events have been shown to elevate both AR and HB-EGF expression in the epidermis (35-37), and our model predicts that cutaneous wounding might lead to increased epidermal expression of AR or HB-EGF and subsequent psoriatic lesion formation at the site of injury, thus explaining on a molecular basis the psoriasis-associated Koebner (isomorphic) phenomena (55).

In summary, this investigation demonstrates clearly that the majority of the clinical and histological features of psoriasis can be reproduced in the mouse within 2-3 wk of age by targeting AR expression to the epidermis. This is in distinct contrast to epidermal TGF- $\alpha$ overexpression, which induces only acanthosis and hyperkeratosis $(41,42)$. Our observations link for the first time the aberrant activity of the epidermal keratinocyte EGF receptor-ligand system to psoriatic inflammation, and also demonstrate that it is possible for defects in keratinocyte physiology to lead to the development of psoriasiform lesions, even in the absence of any primary alteration in the host animal immune system. Results presented here identify future directions for investigation into the molecular and cellular mechanisms mediating the formation and maintenance of psoriasis. Further elucidation of the role of AR and related growth factors (e.g., HB-EGF) in this disease process is warranted, along with characterization of the proinflammatory cytokine networks that are activated in conjunction with overexpression of these keratinocyte-derived autocrine growth factors. In particular, our results suggest that future investigations should also focus on the cellular and molecular basis for the lymphocytic and neutrophilic immigration into the skin of AR-expressing transgenic mice. Finally, the AR-expressing transgenic mouse might also be exploited as a model system to test candidate therapies that block AR binding to its receptor (heparin-like compounds), inhibit the enzymatic activity of EGF receptors (tyrosine kinase inhibitors), or disrupt interactions at points in the signal transduction cascade (Grb2 inhibitors) required for the maintenance of the proliferative signal in epidermal cells. 


\section{Acknowledgments}

The authors wish to thank Norma Swanson, Theodore Trejo, and Karen Squillace for their excellent technical assistance, and Dr. Bruce Cohen (Bristol-Myers Squibb Pharmaceutical Research Institute, Seattle, WA) for generously supplying the 6R1C anti-human AR mAb.

This work was supported in part by the Mayo and J.W. Kieckhefer Foundations (M.R. Pittelkow), the Dermatology Foundation (P.W. Cook), the Collins Medical Trust of Oregon (P.W. Cook), and U.S. Public Health Service grant AR-21557 from the National Institutes of Health (M. Piepkorn).

\section{References}

1. Jegasothy, B.V., C.D. Ackerman, S. Todo, J.J. Fung, K. Abu-Elmagd, and T.E. Starzl. 1992. Tacrolimus (FK 506) - a new therapeutic agent for severe recalcitrant psoriasis. Arch. Dermatol. 128:781-785.

2. Jeffes, E.W. III, J.L. McCullough, M.R. Pittelkow, A. McCormick, J. Almanzor, G. Liu, M. Dang, K. Voss, J. Voss, A. Schlotzhauer, and G.D. Weinstein. 1995. Methotrexate therapy of psoriasis: differential sensitivity of proliferating lymphoid and epithelial cells to the cytotoxic and growth-inhibitory effects of methotrexate. J. Invest. Dermatol. 104:183-188.

3. Lebwohl, M., E. Abel, M. Zanolli, J. Koo, and L. Drake. 1995. Topical therapy for psoriasis. Int. J. Dermatol. 34:673-684

4. Gottlieb, S., P. Gilleaudeau, R. Johnson, L. Estes, T. Woodworth, A. Gottlieb, and J. Krueger. 1995. Response of psoriasis to a lymphocyte-selective toxin (DAB389IL-2) suggests a primary immune, but not keratinocyte, pathogenic basis. Nat. Med. 1:442-447.

5. Lim, K., W. Su, A. Schroeter, C. Sabers, R. Abraham, and M. Pittelkow. 1996. Cyclosporine in the treatment of dermatologic disease: an update. Mayo Clinic Proc. 71:1182-1191.

6. Koo, J., Q. Nguyen, and C. Gambla. 1997. Advances in psoriasis therapy. Adv. Dermatol. 12:47-72.

7. Nickoloff, B.J., G. Gutierrez-Steil, and T. Wrone-Smith. 1997. Dermal injection of CD4+ T cells into symptomless (PN) skin engrafted onto SCID mice induces phenotypic conversion to a psoriatic plaque (PP). J. Invest. Dermatol. 108:539. (Abstr.)

8. Wrone-Smith, T., and B.J. Nickoloff. 1996. Dermal injection of immunocytes induces psoriasis. J. Clin. Invest. 98:1878-1887.

9. Schon, M.P., M. Detmar, and C.M. Parker. 1997. Murine psoriasis-like disorder induced by naive CD4+ T cells. Nat. Med. 3:183-188.

10. Eedy, D.J., D. Burrows, J.M. Bridges, and F.G. Jones. 1990. Clearance of severe psoriasis after allogenic bone marrow transplantation. Br. Med. J. 300: 1398-1399.

11. Gardembas-Pain, M., N. Ifrah, C. Foussard, M. Boasson, J. Saint Andre, and J. Verret. 1990. Psoriasis after allogeneic bone marrow transplantation. Arch. Dermatol. 126:1523.

12. Yin, J.A., and S.N. Jowitt. 1992. Resolution of immune-mediated diseases following allogeneic bone marrow transplantation for leukaemia. Bone Marrow Transplant. 9:31-33.

13. Yokota, A., M. Hukazawa, C. Nakaseko, A. Ishii, T. Ikegami, K. Kogure, M. Nishimura, Y. Matsuura, S. Morio, H. Nakamura, et al. 1996. Resolution of psoriasis vulgaris following allogeneic bone marrow transplantation for aplastic anemia. Rinsho Ketsueki - Jpn. J. Clin. Hematol. 37:35-39.

14. Bata-Csorgo, Z., C. Hammerberg, J.J. Voorhees, and K.D. Cooper. 1995. Kinetics and regulation of human keratinocyte stem cell growth in shortterm primary ex vivo culture. Cooperative growth factors from psoriatic lesional T lymphocytes stimulate proliferation among psoriatic uninvolved, but not normal, stem keratinocytes. J. Clin. Invest. 95:317-327.

15. Shoyab, M., V.M. McDonald, J.G. Bradley, and G.J. Todaro. 1988. Amphiregulin: a bifunctional growth-modulating glycoprotein produced by the phorbol 12-myristate 13-acetate-treated human breast adenocarcinoma cell line MCF-7. Proc. Natl. Acad. Sci. USA. 85:6528-6532.

16. Higashiyama, S., J.A. Abraham, J. Miller, J.C. Fiddes, and M. Klagsbrun. 1991. A heparin-binding growth factor secreted by macrophage-like cells that is related to EGF. Science (Wash. DC). 251:9936-9939.

17. Cook, P.W., P.A. Mattox, W.W. Keeble, M.R. Pittelkow, G.D. Plowman, M. Shoyab, J.P. Adelman, and G.D. Shipley. 1991. A heparin sulfate-regulated human keratinocyte autocrine factor is similar or identical to amphiregulin. Mol. Cell. Biol. 11:2547-2557.

18. Piepkorn, M., C. Lo, and G. Plowman. 1994. Amphiregulin-dependent proliferation of cultured human keratinocytes: autocrine growth, the effects of exogenous recombinant cytokine, and apparent requirement for heparin-like glycosaminoglycans. J. Cell. Physiol. 159:114-120.

19. Cook, P.W., P.A. Mattox, W.W. Keeble, and G.D. Shipley. 1992. Inhibition of autonomous human keratinocyte proliferation and amphiregulin mito- genic activity by sulfated polysaccharides. In Vitro Cell. Dev. Biol. 28A:218222.

20. Cook, P.W., N.M. Ashton, C.E. Karkaria, D.C. Siess, and G.D. Shipley. 1995. Differential effects of a heparin antagonist (hexadimethrine) or chlorate on amphiregulin, basic fibroblast growth factor and heparin-binding EGF-like growth factor activity. J. Cell. Physiol. 163:418-429.

21. Cook, P.W., D. Damm, B.L. Garrick, K.M. Wood, C.E. Karkaria, S. Higashiyama, M. Klagsbrun, and J.A. Abraham. 1995. Carboxyl-terminal truncation converts heparin-binding EGF-like growth factor (HB-EGF) from a heparin-enhancible to a heparin-suppressible growth factor. J. Cell. Physiol. 163: 407-417.

22. Shoyab, M., G.D. Plowman, V.M. McDonald, J.G. Bradley, and G.J. Todaro. 1989. Structure and function of human amphiregulin: a member of the epidermal growth factor family. Science (Wash. DC). 243:1074-1076.

23. Johnson, G.R., T. Saeki, N. Auersperg, A.W. Gordon, M. Shoyab, D.S. Salomon, and M. Stromberg. 1991. Response to and expression of amphiregulin by ovarian carcinoma cells and normal ovarian surface epithelial cells: nuclear localization of endogenous amphiregulin. Biochem. Biophys. Res. Commun. 180:481-488.

24. Higashiyama, S., K. Lau, G.E. Besner, J.A. Abraham, and M.A. Klagsbrun. 1992. Structure of heparin-binding EGF-like growth factor. J. Biol. Chem. 207:6205-6212.

25. Piepkorn, M., R.A. Underwood, C. Henneman, and L.T. Smith. 1995. Expression of amphiregulin is regulated in cultured human keratinocytes and in developing fetal skin. J. Invest. Dermatol. 105:802-809.

26. Piepkorn, M. 1996. Overexpression of amphiregulin, a major autocrine growth factor for cultured human keratinocytes, in hyperproliferative skin diseases. Am. J. Dermatopathol. 18:165-171.

27. Kimura, H. 1993. Schwannoma-derived growth factor must be transported into the nucleus to exert its mitogenic activity. Proc. Natl. Acad. Sci. USA. 90:2165-2169.

28. Shipley, G.D., W.W. Keeble, J.E. Hendrickson, R.J. Coffey, and M.R. Pittelkow. 1989. Growth of normal human keratinocytes and fibroblasts in serum-free medium is stimulated by acidic and basic fibroblast growth factor. $J$. Cell. Physiol. 138:511-518.

29. Cook, P.W., M.P. Pittelkow, and G.D. Shipley. 1991. Growth factorindependent proliferation of normal human neonatal keratinocytes: production of autocrine- and paracrine-acting mitogenic factors. J. Cell. Physiol. 146:277289.

30. Pittelkow, M.R., P.W. Cook, G.D. Shipley, R. Derynck, and R.J. Coffey, Jr. 1993. Autonomous growth of human keratinocytes requires epidermal growth factor receptor occupancy. Cell Growth Differ. 4:513-521.

31. Hashimoto, K., S. Higashiyama, H. Asada, E. Hashimura, T. Kobayashi, K. Sudo, T. Nakagawa, D. Damm, K. Yoshikawa, and N. Taniguchi. 1994. Heparin-binding epidermal growth factor-like growth factor is an autocrine growth factor for human keratinocytes. J. Biol. Chem. 269:20060-20066.

32. Elder, J.T., G.J. Fisher, P.B. Lindquist, G.L. Bennett, M.R. Pittelkow, R.J. Coffey, Jr., L. Ellingsworth, R. Derynck, and J.J. Voorhees. 1989. Overexpression of transforming growth factor $\alpha$ in psoriatic epidermis. Science (Wash. DC). 243:811-814

33. Cook, P.W., M.R. Pittelkow, W.W. Keeble, R. Graves-Deal, R.J. Coffey, and G.D. Shipley. 1992. Amphiregulin messenger RNA is elevated in psoriatic epidermis and gastrointestinal carcinomas. Cancer Res. 52:3224-3227.

34. Hardas, B., Q. Yang, and J.T. Elder. 1992. Regulation of amphiregulin, a heparin-binding, EGF-like growth factor, in human skin. J. Invest. Dermatol. 98:575. (Abstr.)

35. Stoll, S., W. Garner, and J. Elder. 1997. Heparin-binding ligands mediate autocrine epidermal growth factor receptor activation in skin organ culture. J. Clin. Invest. 100:1271-1281.

36. Marikovsky, M., K. Breuing, P.Y. Liu, E. Eriksson, S. Higashiyama, P. Farber, J. Abraham, and M. Klagsbrun. 1993. Appearance of a heparin-binding EGF-like growth factor in wound fluid as a response to injury. Proc. Natl. Acad. Sci. USA. 90:3889-3893.

37. Liou, A., P.M. Elias, C. Grunfeld, K.R. Feingold, and L.C. Wood. 1997. Amphiregulin and nerve growth factor expression are regulated by barrier status in murine epidermis. J. Invest. Dermatol. 108:73-77.

38. King, L.E.J., R.E. Gates, C.M. Stoscheck, and L.B. Nanney. 1990. Epidermal growth factor/transforming growth factor alpha receptors in psoriasis. $J$. Invest. Dermatol. 95:10S-12S.

39. Nanney, L.B., R.A. Yates, and L.E.J. King. 1992. Modulation of epidermal growth factor receptors in psoriatic lesions during treatment with topical EGF. J. Invest. Dermatol. 98:296-301.

40. Elder, J.T., L.-Q. Xia, G. Majmudar, and T. Johnson. 1993. Selective overexpression of amphiregulin in cutaneous squamous and basal cell carcinoma. J. Invest. Dermatol. 100:492. (Abstr.)

41. Vassar, R., and E. Fuchs. 1991. Transgenic mice provide new insights into the role of TGF-alpha during epidermal development and differentiation. Genes Dev. 5:714-727.

42. Dominey, A.M., X.J. Wang, L.E.J. King, L.B. Nanney, T.A. Gagne, K. Sellheyer, D.S. Bundman, M.A. Longley, J.A. Rothnagel, D.A. Greenhalgh, and D.R. Roop. 1993. Targeted overexpression of transforming growth factor alpha in the epidermis of transgenic mice elicits hyperplasia, hyperkeratosis, 
and spontaneous, squamous papillomas. Cell Growth Differ. 4:1071-1082.

43. Guo, L., Q.C. Yu, and E. Fuchs. 1993. Targeting expression of keratinocyte growth factor to keratinocytes elicits striking changes in epithelial differentiation in transgenic mice. EMBO (Eur. Mol. Biol. Organ.) J. 12:973-986.

44. Turksen, K., T. Kupper, L. Degenstein, I. Williams, and E. Fuchs. 1992. Interleukin 6: insights to its function in skin by overexpression in transgenic mice. Proc. Natl. Acad. Sci. USA. 89:5068-5072.

45. Groves, R., T. Rauschmayr, K. Nakamura, S. Sarkar, I.R. Williams, and T.S. Kupper. 1996. Inflammatory and hyperproliferative skin disease in mice that express elevated levels of the IL-1 receptor (type I) on epidermal keratinocytes. Evidence that IL-1-inducible secondary cytokines produced by keratinocytes in vivo can cause skin disease. J. Clin. Invest. 98:336-344.

46. Groves, R.W., H. Mizutani, J.D. Kieffer, and T.S. Kupper. 1995. Inflammatory skin disease in transgenic mice that express high levels of interleukin 1 alpha in basal epidermis. Proc. Natl. Acad. Sci. USA. 92:11874-11888.

47. Cheng, J., K. Turksen, Q. Yu, H. Schreiber, M. Teng, and E. Fuchs. 1992. Cachexia and graft-vs.-host-disease-type skin changes in keratin promoter-driven TNF alpha transgenic mice. Genes Dev. 6:1444-1456.

48. Carroll, J.M., T. Crompton, J.P. Seery, and F.M. Watt. 1997. Transgenic mice expressing IFN- $\gamma$ in the epidermis have eczema, hair hypopigmentation, and hair loss. J. Invest. Dermatol. 108:412-422.

49. Williams, I.R., R.J. Ort, and T.S. Kupper. 1994. Keratinocyte expression of B7-1 in transgenic mice amplifies the primary immune response to cutaneous antigens. Proc. Natl. Acad. Sci. USA. 91:12780-12784.

50. Williams, I.R., and T.S. Kupper. 1994. Epidermal expression of intercellular adhesion molecule 1 is not a primary inducer of cutaneous inflammation in transgenic mice. Proc. Natl. Acad. Sci. USA. 91:9710-9714.

51. Blessing, M., P. Schirmacher, and S. Kaiser. 1996. Overexpression of bone morphogenetic protein-6 (BMP-6) in the epidermis of transgenic mice: inhibition or stimulation of proliferation depending on the pattern of transgene expression and formation of psoriatic lesions. J. Cell Biol. 135:227-239.

52. Carroll, J.M., M.R. Romero, and F.M. Watt. 1995. Suprabasal integrin expression in the epidermis of transgenic mice results in developmental defects and a phenotype resembling psoriasis. Cell. 83:957-968.

53. Vassar, R., M. Rosenberg, S. Ross, A. Tyner, and E. Fuchs. 1989. Tissue-specific and differentiation-specific expression of a human K14 keratin gene in transgenic mice. Proc. Natl. Acad. Sci. USA. 86:1563-1567.

54. Plowman, G.D., G.S. Whitney, J.M. Green, V.L. McDonald, M.G. Neubauer, C.M. Disteche, G.J. Todaro, and M. Shoyab. 1990. The amphiregulin gene encodes a novel epidermal growth factor-related protein with tumorinhibitory activity. Mol. Cell. Biol. 10:1969-1981.

55. Pittelkow, M.R. 1991. Keratinocyte abnormalities. In Psoriasis. H.H. Roenigk and H.I. Maibach, editors. Marcel Dekker, Inc., New York. 305-325.

56. Wright, A.L., and A.G. Messenger. 1990. Scarring alopecia in psoriasis. Acta Dermato-Venereol. 70:156-159.

57. Runne, U., and P. Kroneisen-Wiersma. 1992. Psoriatic alopecia: acute and chronic hair loss in 47 patients with scalp psoriasis. Dermatology (Basel) 185:82-87.

58. Schoorl, W.J., H.J. van Baar, and P.C. van de Kerkhof. 1992. The hair root pattern in psoriasis of the scalp. Acta Dermato-Venereol. 72:141-142.

59. Headington, J.T., A.K. Gupta, M.T. Goldfarb, B.J. Nickoloff, T.A. Hamilton, C.N. Ellis, and J.J. Voorhees. 1989. A morphometric and histologic study of the scalp in psoriasis. Paradoxical sebaceous gland atrophy and decreased hair shaft diameters without alopecia. Arch. Dermatol. 125:639-642.

60. Runne, U., and P. Kroneisen. 1989. Psoriatic alopecia manifestation, course and therapy in 34 patients. Z. Hautkr. 64:302-304.

61. Shuster, S. 1972. Psoriatic alopecia. Br. J. Dermatol. 87:73-77.

62. Philpott, M., D. Sanders, G. Westgate, and T. Kealey. 1994. Human hair growth in vitro: a model for the study of hair follicle biology. J. Dermatol. Sci. 7: S55-S72.

63. Coulombe, P.A., R. Kopan, and E. Fuchs. 1989. Expression of K14 in the epidermis and hair follicle: insights into complex programs of differentiation. J. Cell Biol. 109:2295-2312.

64. Byrne, C., M. Tainsky, and E. Fuchs. 1994. Programming gene expression in developing epidermis. Development (Camb.). 120:2369-2383. 